REVISTA CIENCIAS BIOMÉDICAS

ARTÍCULOS ORIGINALES

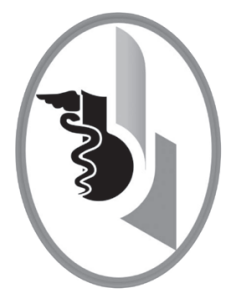

\title{
DESENSIBILIZACIÓN Y REPROCESAMIENTO POR MEDIO DE MOVIMIENTO OCULAR (EMDR): UNA TERAPÉUTICA PARA EL ESTRÉS POSTRAUMÁTICO
}

\author{
EYE MOVEMENT DESENSITIZATION AND REPROCESSING \\ (EMDR): TREATMENT FOR POSTTRAUMATIC STRESS \\ DISORDER
}

Navas Torrejano Diana Stefanía ${ }^{1}$

Correspondencia: dnavast@unicartagena.edu.co

Recibido para evaluación: noviembre - 16 - 2010. Aceptado para publicación: Marzo - 15- 2011

\section{RESUMEN}

El trastorno de estrés postraumático está clasificado como uno de los trastornos de ansiedad dado como una respuesta patológica a un evento estresante que supone un riesgo físico o psicológico. Corresponde a un problema de salud pública que causa gran incapacidad y dificultades en el desarrollo biopsicosocial de la persona afectada. Actualmente se llevan a cabo diferentes métodos terapéuticos para el tratamiento de dicha patología, dentro de las mas estudiadas y con amplios resultados positivos se encuentra la terapia de reprocesamiento llamada Desensibilización y Reprocesamiento por medio de Movimiento Ocular (EMDR por sus siglas en inglés) basado en estimulación bilateral ocular, principalmente, que otorga al paciente la oportunidad de asimilar el evento traumático transformando su contenido emocional y brindando adaptación e integración de la información y equilibrio físico y psicológico con respuestas adaptativas que permite el desarrollo e interacción normal con el entorno. Con la Técnica EMDR para enfrentar el trastorno de estrés postraumatico, se alcanza que si bien el recuerdo está, ya no hiere. Rev.cienc.biomed.2011; 2 (1): 158-162

\section{PALABRAS CLAVES}

EMDR. Estrés Postraumático. Reprocesamiento.

\section{SUMMARY}

Posttraumatic stress disorder is classified as an anxiety disorder characterized for a pathological response to a stressful event that involves a physical or psychological risk. It is a public health problem that causes great disability and difficulties in biopsychosocial development of the patient. Currently, there are different therapeutic methods for treating this disease; the most studied one with positive results is "eye movement desensitization and reprocessing" (EMDR) based on bilateral visual stimulation, which gives the patient an opportunity to assimilate the traumatic event, transforming its emotional content and providing adaptation and integration of information and physical and psychological balance with adaptive responses allowing normal development and interaction with the environment. With EMDR to address post-traumatic stress disorder the memory is there, but it does not hurt. Rev.cienc.biomed. 2011,2 (1): 158162

\section{KEYWORD}

EMDR. Posttraumatic stress disorder. Reprocessing. 
El Trastorno de estrés postraumático (TEPT) es una condición clínica que representa una de las respuestas psíquicas a un evento traumático. Fue incluido de manera oficial en el manual diagnóstico estadístico de desordenes mentales alrededor de 1980 en su tercera versión. Está clasificado dentro del grupo de los trastornos de ansiedad, que sobreviene como consecuencia de la exposición a un evento traumático que involucra un daño o perdida de la integridad personal física o psicológica. Es una severa reacción emocional a un trauma psicológico extremo, un grado tal, que las defensas mentales de la persona no pueden asimilarlo. El término TEPT engloba dos aspectos bien definidos: por una parte una respuesta patológica de estrés, y por otra el trauma. Este diagnóstico constituye un problema de salud pública por su alta prevalencia, por afectar de preferencia a población activa, por presentarse tanto en población general como en combatientes, por la incapacidad que genera y por los altos costos de salud que implica. Es un cuadro que en general ha sido subdiagnosticado y por ende subtratado. En los últimos 25 años, más de 150 millones de personas anualmente son afectadas directamente por diferentes tipos de desastres y acontecimientos traumáticos según las diferentes condiciones.

Los efectos físicos son evidentes. Miles de personas pierden sus vidas o son gravemente heridas, los sobrevivientes arrastran las consecuencias durante toda su vida: dolor y sufrimiento se distribuyen a partes iguales; los efectos emocionales: miedo, ansiedad, estrés, ira, rabia, resentimiento y bloqueo emocional.

La respuesta de estrés de los pacientes con TEPT no corresponde a una exageración de la respuesta normal, sino que incluye diversos indicadores biológicos que permiten caracterizar e individualizar la respuesta específica de este cuadro clínico. EI TEPT se caracteriza por una tríada sintomática de fenómenos invasores, conductas de evitación y síntomas de hiperalerta en respuesta a un acontecimiento traumático, el cual puede presentarse en cualquier sujeto y a cualquier edad. El término TEPT engloba dos aspectos bien definidos: por una parte una respuesta de estrés que naturalmente es patológica, y por otra el trauma. Estrés es un concepto científico que alude a una respuesta inespecífica del organismo ante una demanda; frente a un estresor físico o psicológico, el organismo responderá buscando la adaptación y el equilibrio (homeostasis) la respuesta puede resultar adaptativa. Lo cual es el caso de muchas víctimas, en las que estos efectos se mitigan e incluso desaparecen con el tiempo (eustrés). Sin embargo, para otros muchos, las secuelas son a largo plazo y alcanzan en ocasiones la condición de crónicas si no reciben el tratamiento adecuado, y provoca un desajuste consigo mismo y el entorno que lo rodea (distrés). Los mecanismos de defensa que inicialmente protegen al abusado, progresivamente se vuelcan contra él, impidiendo la recuperación y el cambio a mecanismos adaptativos, llevando a un deterioro de las habilidades manifestadas en disminución de la atención, memoria, hipervigilancia, flashbacks todo con disminución en la calidad de vida y la relación con el entorno. Por ello, es importante que a estas personas se les brinde ayuda integral incluyendo el área psicológica.

\section{EMDR: EYES MOVEMENT DESENSITI- ZATION AND REPROCESSING}

En 1987 fue propuesta por la Doctora Francine Shapiro y desde entonces se ha venido estudiando una nueva terapia basada en métodos fisiológicos de reprocesamiento de las experiencias, llamado EMDR (Desensibilización y Reprocesamiento a través de Movimientos Oculares) que ha demostrado ser muy útil en el tratamiento de experiencias traumáticas, incluyendo el abuso sexual. Dicha técnica se orienta en la desensibilización a la ansiedad y da lugar a un nuevo paradigma que considera el procesamiento de la información y las redes asociativas, asimismo plantea la existencia de un sistema inherente en toda persona, sistema que está fisiológicamente preparado para procesar información hasta que esta lleve a una resolución adaptativa, que no es más que un estado de salud mental que implica que las emociones negativas se eliminan y hace que la información se encuentre en un futuro de forma integrada. 


\section{BASES NEUROFISIOLÓGICAS}

La teoría más prominente para explicar el éxito de la terapia tiene que ver precisamente con los movimientos oculares que se llevan a cabo durante el sueño REM, fase donde se procesan las experiencias del día y la relación de esta con la corteza y el sistema límbico. Normalmente las experiencias vividas con carga emocional significativa son recopiladas en los cuerpos amigdalinos y al recordarse se reproduce con las sensaciones y emociones que lo acompañaban, esta estructura se activa durante el sueño para modular y almacenar y asimilar las vivencias en la corteza prefrontal izquierda y el hipocampo, permitiendo que el individuo pueda utilizarlas para enfrentar eventos en el futuro.

Durante situación de estrés severo, la excesiva estimulación de la amígdala interviene con la función reguladora de la corteza y el hipocampo, con lo que el suceso es almacenado como un conjunto de sensaciones no procesado ni contextualizado en espacio y tiempo. Consecuentemente se observa una inhibición de la corteza izquierda y sobreactivación de la derecha al tiempo que debido al aumento de norepinefrina se inhiben las neuronas colinérgicas pedúnculopontinas del tegmento que generan el sueño REM, lo cual, según se postula, impide que se procesen adecuadamente los recuerdos traumáticos creando un nudo neural con recuerdos "congelados" que pueden ser activados ante cualquier situación gatillo y causa una serie de síntomas típicos del stress postraumático.

\section{EN QUÉ CONSISTE LA TERAPIA}

EMDR es una técnica innovadora, un modelo de Psicoterapia complejo que integra elementos y componentes exitosos de diferentes enfoques basado en los movimientos oculares rápidos que se llevan a cabo en la fase del sueño REM durante la cual se realiza precisamente la integración de las experiencias diarias en las redes neuronales desde la información proveniente de los sentidos y su significado emocional por activación de los cuerpos amigdalinos hasta su modulación por la corteza prefrontal izquierda y la contextualización de la misma en el hipocampo. Es hasta ahora la terapia mas fisiológica ya que incorpora una simulación de lo que ocurre durante el sueño REM pero de manera consciente y orientada, con estimulación bilateral que puede ser además de la ocular (con la cual se ha tenido la mejor respuesta), sonora o táctil en conjunto con terapia cognitivo-conductual, psicodinamia, terapia bioinformacional, entre otras, de manera que se pueda revivir las partes más perturbadoras del trauma y modificar las actitudes hacia el mismo sin el riesgo de ser interrumpidas, como sucede con las pesadillas; modulando la información, integrándola y proporcionando un equilibrio integral en la persona afectada. Cada sesión consta de seis fases dentro de las cuales se da un periodo de preparación, evaluación, desensibilizacion, instalación, escáner y cierre con una reevaluación posterior, trabajando con las experiencias traumáticas pasadas, las situaciones presentes que despiertan dichos recuerdos y los patrones futuros de acciones apropiadas. El procedimiento se repite hasta llevar a la memoria por todas las ramificaciones y generalizaciones que se han dado a raíz del incidente por un sendero adaptativo, logrando así balancear nuevamente el sistema ya que de lo contrario en el futuro pueden convertirse en el agente disparador elucidando toda la sintomatología antes presente.

\section{RESULTADOS}

Los resultados terapéuticos de EMDR se relacionan con el hallazgo de que a través de los movimientos oculares y el reprocesamiento de la información se reduce la vivencia de las imágenes estresantes, esto relacionado con cambios en la función viso espacial y la memoria de trabajo que hacen que se reduzca la intensidad de la emoción asociada a la imagen y el recuerdo traumático. Algunos estudios demuestran que después del tratamiento con EMDR los recuerdos tanto positivos como negativos se hacen menos "vividos", es decir tienen una carga emocional menor.

Algunos de los cambios en imágenes como la tomografía por emisión de positrones (SPECT) descritos después del tratamiento con EMDR incluyen hiperactividad del cíngulo anterior y el lóbulo frontal izquierdo, este hallazgo señala que el tratamiento aumenta 
la actividad del sistema límbico lo que termina mejorando la capacidad del individuo para diferenciar una amenaza real de una imaginaria. Electroencefalográficamente se ha evidenciado la normalización de las ondas cerebrales más lentas en ambas cortezas cerebrales, esto sugiere la resincronización de la actividad de ambos hemisferios. En un reciente estudio preliminar utilizando tomografía computarizada por emisión de fotones simples, se ha observado que ciertos movimientos físicos oculares y de otros músculos, en estado de vigilia, activan las células tegmentales antedichas, que generan sueño MOR.

\section{EVIDENCIAS}

Este tema ha suscitado un largo y apasionado debate acerca de su cientificidad. Las investigaciones de los últimos 20 años convierten al EMDR en uno de los tratamientos con mayores estudios de validación científica y con alta eficacia en el tratamiento del Estrés Postraumatico.

En uno de ellos realizado con 60 mujeres jóvenes traumatizadas que fueron divididas aleatoriamente en dos grupos: un grupo bajo terapia EMDR y otro grupo control se analizaron con ANOVA los resultados dados por diferentes pruebas (Inventario de Depresión de Beck, Inventario de Ansiedad de EstadoRasgo, Inventario de Penn para TEPT, Escala de Impacto de Eventos, Escala de Autoconcepto de Tennessee) y se encontró una mejoría significativa en ambos grupos, pero esta recuperación fue significativamente mayor en el grupo tratado con EMDR: 0.65 en el grupo control y 1.56 en el grupo experimental. Después del tratamiento, las medias de todas las variables del grupo tratado con EMDR fueron comparables con las de control u otros grupos tratados exitosamente.

En otro estudio realizado por Marcus et al se repartieron aleatoriamente a 67 personas con TEPT en dos grupos. Uno recibió tratamiento con EMDR y el otro cuidado estándar. Se utilizaron como técnicas para medir los resultados: Lista de Síntomas-90, Inventario de Depresión de Beck, Escala de Impacto de Eventos, Escala modificada de TEPT, Inventario de Ansiedad Estado-Rasgo de Spielberger y
Unidades Subjetivas de Perturbación; además, un evaluador externo utilizó los criterios del DSM-III-R. El grupo experimental mostró una mejoría significativamente mayor que el grupo control y con mucha rapidez, en todas las medidas de TEPT, depresión, ansiedad y síntomas generales.

En una investigación con veteranos de guerra, se dividieron al azar 35 de ellos en tres grupos y se manejaron así: el primero, 12 sesiones de EMDR; el segundo, 12 sesiones de relajación biorretroalimentada y el tercero, cuidado clínico rutinario. Se realizaron varias pruebas de autoevaluación, psicométricas y entrevistas estandarizadas. Los efectos de EMDR fueron significativamente mayores que los otros dos, y tres meses después permanecían.

Recientemente, "Department of Defense" y el "Department of Veteran Affairs" de los Estados Unidos han publicado nuevas pautas que ubican a EMDR en la categoría de terapias con el más alto nivel de evidencia y la recomiendan como uno de los tratamientos de elección para el manejo del TEPT. De igual manera ha sido avalada por International Society for Traumatic Stress Studies, The United Kingdom Department of Health, the American Red Cross, the FBI, the International Critical Incident Stress Management Foundation, and Major HMO's such as Kaiser and Value Options y American Psychological Association Division of Clinical Psychology's list, en la que solo otros dos métodos son incluídos, además del EMDR.

Estudios de seguimiento a 5 años confirman la eficacia de la técnica con mantenimiento de los efectos del tratamiento sobre TEPT por cualquier causa. No obstante, se ha intentado utilizar EMDR en otro tipo de pacientes con trastornos de ansiedad, fobias, trastornos alimentarios y aunque aún no puede concluirse con seguridad su efectividad, los primeros estudios indican que es poca.

A pesar de que no hay tantos estudios controlados como en los demás grupos etáreos, la EMDR se ha encontrado tan eficaz en los niños como en los adultos y además con mayor rapidez en las respuestas adaptativas. Se ha postulado para el tratamiento de asuntos 
como el maltrato infantil, la violencia y acceso carnal violento, que provocan el desvalimiento o la impotencia en el niño. Actualmente, los terapeutas utilizan EMDR en niños incluso desde los dos años de edad, convirtiendo niños potencialmente desadaptados por eventos trágicos no asimilados en personas con capacidad de sobrellevar su vida, su condición y sus experiencias con mayor madurez y mejores respuestas acondicionadas.

\section{CONCLUSIÓN}

La terapia EMDR es un método no invasivo, relativamente simple y altamente efectivo para la resolución del trastorno de estrés postraumático cuyos fundamentos fisiológicos han sido demostrados con métodos de evaluación objetivos que la avalan científicamente y la proponen como una de las terapias de elección para el manejo de dicha patología por cualquier causa, en cualquier grupo etáreo con los mismos resultados. Proveyendo una reestructuración de las experiencias vividas y la contextualización de las mismas, hasta lograr que sean percibidos de manera positiva o neutra, menos vívidas y menos válidas, lo cual permite al individuo una asimilación y producción de respuestas adaptativas futuras.

CONFLICTOS DE INTERÉS: ninguno que declarar.

FINANCIACIÓN: recursos propios del autora.

\section{LECTURAS RECOMENDADAS}

1. Camacho G. Desensibilización y reprocesamiento por movimientos oculares (EMDR) Artículos Medicina Integrativa. Abril 2010

2. Carvajal C. Trastorno por estrés postraumático: aspectos clínicos. Rev. chil. neuropsiquiatr. 2002; 40 (2): 717-727

3. Escamilla M. Colombia aprende. Cartilla. Ministerio de Protección Social. Bogotá. 2010.

4. Gil Lemu L. Desensibilización y reprocesamiento con movimientos oculares. rev.colomb. psiquiatr 2008; 37(1): $5-12$.

5. Lovett J. La Curación Del Trauma Infantil Mediante Drmo. Rústica (1). 2000

6. Parnell, L. Transforming Trauma: EMDR, the revolutionary new therapy for freeing the mind, clearing the body and opening the heart. NY: W.W Norton@Co. (1997)

7. Shapiro F. EMDR. Desensibilizacion y Reprocesamiento por medio de Movimiento Ocular. Espasa Calpe. Pax Mexico 2004; 439 (270-1): 968-860

8. Shapiro F. Eye Movement Desensitization and Reprocessing (EMDR) and the anxiety disorders: clinical and research implications of an integrated psychotherapy treatment. J Anxiety Disord. $1999 ; 13(1-2): 35-67$

9. Scholten, A. Desensibilización y Reprocesamiento por Movimiento Ocular: Un Tratamiento Controversial para Los Sobrevivientes de Trauma. EBSCO publishing 2009. Disponible en: http://www.mbmc.org/healthgate

10. Silverton M. EMDR - A Powerful Tool for Healing. Epping Forest Road, Annapolis. 2010 Disponible en: www.rcshelpingcouples.com/PDFs/ToolForHealing.pdf

11. Trejos-Parra JJ, Betancur-Sánchez AV, Montoya-Alzate LS. Desensibilización y reprocesamiento mediante movimientos oculares: terapia efectiva para el trastorno por estrés postraumático. 2008. URL disponible en: www.amauta-international.com/ArtRevMDRis.htm

12. What is EMDR? Dr Kenney has been trained in the use of EMDR with adults, adolescents and children. Blooming Prairie Assessment \& Therapy Center. 2010 Disponible en: www. bloomingprairiecenter.com/what_is_emdr 